\section{Immuntherapie spart Glukokortikoide ein}

$B^{\mathrm{c}}$ ei Kindern mit allergischem Asthma bronchiale aufgrund einer Sensibilisierung gegen Hausstaubmilben wird gemäß den Leitlinien der Global Initiative for Asthma (GINA) eine Dauertherapie mit inhalativen Glukokortikoiden bereits bei geringgradig persistierenden Beschwerden empfohlen. Allerdings sollte darauf geachtet werden, die Glukokortikoide so niedrig wie möglich zu dosieren.

Inwieweit die subkutane Immuntherapie (SCIT), die beim Indikationsgebiet allergische Rhinokonjunktivitis gut etabliert ist, beim durch Hausstaubmilbenallergene ausgelösten Asthma bronchiale wirkt und zur Verringerung der Glukokortikoiddosis beiträgt, wurde nun in einer kontrollierten Multizenterstudie bei Kindern und Jugendlichen untersucht (Zielen $S$ et al. J Allergy Clin Immunol 2010; 126: 942-9). Zur Hyposensibilisierung wurde das hypoallergene Hochdosispräparat Acaroid $^{\circledR}$ eingesetzt, welches sich nicht nur durch seine Effektivität, sondern auch durch seine gute Verträglichkeit auszeichnet. Acaroid ${ }^{\circledR}$ ist für Kinder $a b$ sechs Jahren geeignet.

An der Studie beteiligt waren 65 Kinder zwischen sechs und 17 Jahren mit durch Hausstaubmilben ausgelöstem allergischen Asthma und einem täglichen Bedarf an inhalativen Glukokortikoiden von mindestens $2 \times 50 \mu \mathrm{g}$ Fluticasonpropionat (FP). Sie erhielten über zwei Jahre hinweg randomisiert entweder eine SCIT mit Acaroid ${ }^{\circledR}$ plus FP oder ausschließlich die antiasthmatische Basis- und Bedarfsmedikation.

Die zur Symptomkontrolle erforderliche minimale FP-Dosis konnte bei den Patienten unter der SCIT im Vergleich zur Kontrollgruppe im Verlauf der The-

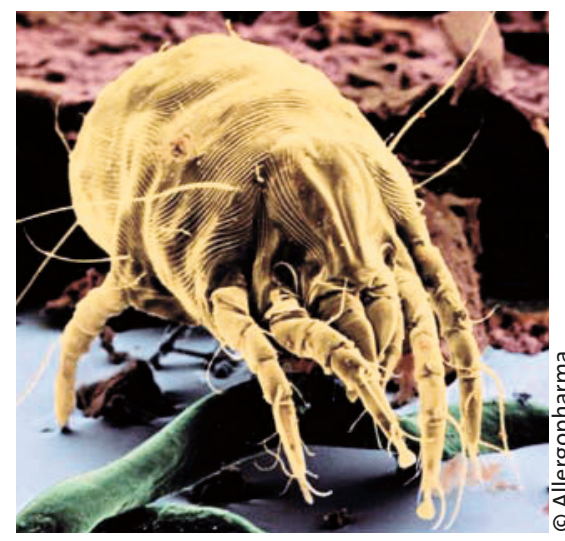

Um ein durch Hausstaubmilben ausgelöstes Asthma bronchiale bei Kindern unter Kontrolle zu bringen, sind nach einer spezifischen Immuntherapie deutlich weniger Glukokortikoide erforderlich. rapie signifikant reduziert werden $(\mathrm{p}<$ $0,05)$. Die tägliche Glukokortikoiddosis konnte durch die SCIT nach zwei Jahren mehr als halbiert werden (um 53\% von $330,3 \mu \mathrm{g}$ auf $151,5 \mu \mathrm{g}$ FP). In der Kontrollgruppe sank der Bedarf kaum. Trotz der geringeren Glukokortkoidgaben blieb das Asthma in der Verumgruppe gut kontrolliert, die Lungenfunktion im morgendlichen Peak Flow besserte sich sogar signifikant gegenüber der Kontrollgruppe und die unspezifische bronchiale Hyperreagibilität verschlechterte sich nicht. Die IgG1- und insbesondere die IgG4-Antikörper stiegen unter der Acaroid $^{\circledR}$-Therapie deutlich an - ein Beleg für die Aktivität des standardisierten Milbenpräparats auf immunologischer Ebene.

Da nicht nur die Wirksamkeit der hypoallergenen Hochdosis-SCIT gut war, sondern auch die Verträglichkeit, empfehlen die Autoren der Studie diese Therapieoption als sichere und effektive Behandlungsoption. Die Daten der Studienpopulation werden aktuell noch weiter verfolgt mit dem Ziel, den aus anderen Studien bekannten Langzeiteffekt der SCIT auch bei Kindern mit durch Hausstaubmilben ausgelöstem Asthma nachzuweisen.

red

Nach Informationen von Allergopharma, Reinbek

\title{
Fünf-Gräser-TabletteimDrei-Jahres-Test
}

W irksamkeit und Sicherheit der Fünf-Gräser-Tablette Oralair $^{\circledR}$ bei der Therapie von gräserpollenbedingten Atemwegserkrankungen sind durch zwei große doppelblind-plazebokontrollierte Studien mit Erwachsenen, Jugendlichen und Kindern ab fünf Jahren belegt. Nun liegen die ersten Ergebnisse zur Langzeitwirksamkeit über drei Jahre vor, referierte Prof. Dr. Christian Virchow, Rostock. Er berichtete über eine multizentrische, randomisierte und plazebokontrollierte Doppelblindstudie mit 633 erwachsenen Patienten mit allergischer Rhinitis aufgrund einer Sensibilisierung gegen Gräserpollen. Während der drei prä- und kosaisonalen Behand- lungszyklen gingen die Symptome durch die Hyposensibilisierung kontinuierlich zurück. In der dritten Pollensaison lag die mediane Differenz zwischen Verum und Plazebo bei 50\%, gemessen anhand des durchschnittlichen adjustierten Symptomscores (AAdSS). Bei diesem von den Zulassungsbehörden anerkannten primären Studienendpunkt wird die allergische Symptomatik von Nase und Auge am Medikamentenverbrauch des Vortages adjustiert. „Die Zahl der Studienabbrecher war über die drei Jahre insgesamt sehr gering", ergänzte Virchow ein wichtiger Hinweis auf die gute Therapieadhärenz und die Verträglichkeit der Behandlung.
Die zunehmende Evidenz zur Wirksamkeit und Sicherheit der Tablettentherapie werde sich sicherlich auch in der nächsten deutschen Leitlinie zur spezifischen Immuntherapie niederschlagen, prophezeite Prof. Dr. Claus Bachert, Gent, Belgien: „Aufgrund der jetzt vorliegenden großen Studien vor allem mit Allergentabletten hat die sublinguale die subkutane Immuntherapie bei der Evidenz bereits überholt." Auch müsse man sich mehr als bisher um eine produktspezifische Bewertung bemühen.

Lunch-Symposium „Mehr Evidenz in der Spezifischen Immuntherapie“ im Rahmen der 22. Fortbildungswoche für praktische Dermatologie und Venerologie, München, 28. Juli 2010. Veranstalter: Stallergenes, Kamp-Lintfort 